\author{
Annual Report for \\ Contract No. DE-FG02-91ER40623 \\ in the 1991-1992 Year
}

\title{
Research in High Energy Physics
}

\author{
J. LoSecco
}

\author{
Physics DePARTMENT \\ UNIVERSITY OF NOTRE DAME \\ Notre DAme, INDIANA 46556 USA
}

\begin{abstract}
Progress in the operation of the 8000 ton IMB detector is reported. During the last year there has been an emphasis on extracting physics results from the 1986-1988 data sample. Analysis of the 1989-90 data sample has also picked up. The Notre Dame group has contributed greatly to tise operation, organization and dissemination of physics results from IMB. We have also played a key role in attempting to preserve the detector and detector site for possible future ventures.

Activities in Brookhaven Experiment 852, a search for mesons with unusual quantum numbers has increased this year. We are currently in the design and building phase of this project which i: scheduled to begin data taking in early 1993.

Our Notre Dame group is also exploring possibilities for smaller SSC experiments which may become feasible in the near future.
\end{abstract}

\section{MASTER}




\section{Contents}

1 Introduction 4

2 Physics with IMB

2.1 Introduction . . . . . . . . . . . . . . . 4

2.2 Proton Decry . . . . . . . . . . . . . . . 5

2.3 Point Source Search . . . . . . . . . . . . . . . . 6 6

2.4 Underground Muons from Cygnus X3 .......... 9

2.5 Dark Matter . . . . . . . . . . . . . . . . . . . . 11

2.6 Neutrino Oscillations . . . . . . . . . . . . . 12

2.7 The Future of IMIB . . . . . . . . . . . . . . . . 14

3 Meson Spectroscopy $\quad 15$

3.1 Physics Motivation ................ 1.5

3.2 Brookaven E852 ................ . . 16

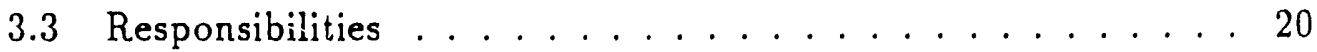

3.4 Progress to Date . . . . . . . . . . . . . . 20

\section{DISCLAIMER}

This report was prepared as an account of work sponsored by an agency of the United States Government. Neither the United States Government nor any agency thereof, nor any of their employees, makes any warranty, express or implied, or assumes any legal liability or responsibility for the accuracy, completeness, or usefulness of any information, apparatus, product, or process disclosed, or represents that its use would not infringe privately owned rights. Reference herein to any specific rommercial product, process, or service by trade name, trademark, manufacturer, or otherwise Jnes not necessarily constitute or imply its endorsement, iecommendation, or favoring by the United States Government or any agency thereof. The views and opinions of authors expressed herein do not necessarily state or reflect those of the United States Government or any agency thereof. 


\section{Introduction}

We review the efforts of our Notre Dame high energy physics group. Dur efforts in this period has been directed toward the IMB deep underground detector and E852 a Brookhaven meson spectroscopy experiment. Our formal responsibilities in IMB include diver maintenance and safety, processing a large share of the data, online computing and several physics objectives.

Currently we are studying proton decay, foint neutrino sources, the Cygnus X3 summer 1989, 1990 and January 1991 radio outburst, sources of dark matter and neutrino oscillations.

Our work in meson spectroscopy has been in designing and implementing the apparatus for a run in early 1993. The device involves modifications to the MPS to study all neutral final states. This program will become our dominant effort during the next contract periud.

\section{Physics with IMB}

\subsection{Introduction}

We continue to extract physics results from the IMB data sample. While the full final data sample is not available we do have the worlds largest sample of contained neutrino interactions and upward going muons from muon neutrino interactions. We have been working with the combined 887 event IMB 1 plus IMB 3 data sample which come from 793 live days of operation and 651 upward going muons collected in IMB 12 and 3 from about 1200 live days.

There have been persistent claims of undergiound muons associated with radio flares of Cygnus $\mathrm{X} 3$. We have stepped into the role of checking our through going downward muons against this hypothesis whenever we learn of a possible radio outburst. Outbursts were reported in 1989,1990 and 1991. We never intended to be the responsible party on this subject for IMB but we stepped in when claims were advertised outside the group even though the data had not been analyzed.

While our primary physics objective remains proton decay. Most effort this period has been dedicated to neutrino physics and what can be learned 
Table 1. Nucleon Decay Lifetimes (years)

\begin{tabular}{|c|c|c|c|c|c|c|}
\hline mode & eff & $\begin{array}{c}\text { no. } \\
\text { cand }\end{array}$ & $\begin{array}{c}\text { no. } \\
\text { bckgnd }\end{array}$ & $\begin{array}{c}\text { lifetime } \\
\text { (years) }\end{array}$ & $\begin{array}{l}\text { bckgrnd } \\
\text { subtrctd }\end{array}$ & PDB \\
\hline$p \rightarrow e^{+} \pi^{11}$ & .42 & 1 & 0.4 & $9.6 \times 10^{.31}$ & $9.6 \times 10^{311}$ & 24 \\
\hline$p \rightarrow \mu^{+} \pi^{\prime \prime}$ & .36 & 0 & 0.2 & $1.4 \times 10^{32}$ & $1.4 \times 10^{132}$ & $2 . \bar{T}$ \\
\hline$p \rightarrow \mu^{+} \gamma$ & .62 & 0 & 0.2 & $2.5 \times 10^{32}$ & $2.5 \times 10^{132}$ & 3.8 \\
\hline$p \rightarrow e^{+} \gamma$ & .63 & 1 & 0.4 & $1.4 \times 10^{32}$ & $1.4 \times 10^{32}$ & 4.6 \\
\hline$n \rightarrow e^{+} \pi^{-}$ & .15 & 0 & 0.4 & $4.8 \times 10^{31}$ & $4.8 \times 10^{: 31}$ & 13. \\
\hline$n \rightarrow \mu^{+} \pi^{-}$ & .25 & 2 & 0.7 & $3.4 \times 10^{.31}$ & $4.6 \times 10^{: 31}$ & 10. \\
\hline$p \rightarrow \mu^{+} \eta(\eta \rightarrow \gamma \gamma)$ & .11 & 0 & 0.4 & $3.1 \times 10^{31}$ & $3.1 \times 10^{: 31}$ & 6.9 \\
\hline$p \rightarrow \mu^{+} \omega\left(\omega \rightarrow \pi^{\prime \prime} \gamma\right)$ & .46 & 1 & 0.6 & $8.8 \times 10^{: 311}$ & $1.6 \times 10^{: 31}$ & $5 . \overline{1}$ \\
\hline$p \rightarrow \mu^{+} \rho\left(\rho \rightarrow \pi^{+} \pi^{-}\right)$ & .20 & 3 & 1.5 & $2.7 \times 10^{31}$ & $4.1 \times 10^{.11}$ & 11. \\
\hline$p \rightarrow \mu^{+} \mu^{+} \mu^{-}$ & .65 & 3 & 1.3 & $8.8 \times 10^{331}$ & $1.1 \times 10^{132}$ & 1.9 \\
\hline$p \rightarrow e^{+} e^{+} e^{-}$ & .33 & 1 & 0.4 & $7.8 \times 10^{311}$ & $7.8 \times 10^{: 31}$ & 51. \\
\hline$p \rightarrow \mu^{+} K_{0}^{\prime \prime}\left(K_{0}^{\prime \prime} \rightarrow \pi^{+} \pi^{-}\right)$ & .46 & 5 & 3.1 & $4.6 \times 10^{31}$ & $7.9 \times 10^{: 31}$ & 12 \\
\hline
\end{tabular}

with neutrinos.

\subsection{Proton Decay}

Most of the work associated with calculating limits on proton decay involves simulations of the many different decay modes and simulations, perhaps more than one, of the anticipated background. We at Notre Dame have prepared such computer files for IMB 3 as far back as 5 years ago. The rest of the work is to study the simulations to develop cuts that will maximize a signal, as characterized by the decay simulations, while minimizing the background.

At Notre Dame we have carried this out twice. Our initial effort produced a large list of limits on proton decay using the classic energy-anisotropy method. The method is very general and works for most decay modes. The anisotropy is a rough measure of the unbalanced momentum in an event. The energy can be compared with the expected energy deposition from a true proton decay. The most serious failing of this method is that it is not very good at rejecting background. The detector does give more information than these two variables and that information is discarded with this technique. Still it is the only effort, to date, to study all possible proton decays with 
Table 2: Contained Event Point Source Search 887 Events input. 59.41 expected per source

\begin{tabular}{|c|c|c|c|c|c|c|c|}
\hline & Right & & Event & Spect. & Rate & Peaking & $90 \%$ \\
\hline & Ascen & Decl. & Count & Prob & Prob & Prob & \\
\hline LMC.X4 & 5.5 & -66 & 53 & .126 & .814 & .114 & 10.4 .1 \\
\hline CENT A & 13.4 & -43 & $50 \hat{~}$ & .773 & .689 & .023 & 11.87 \\
\hline VELA X1 & 9.0 & .41 & 66 & .107 & .213 & .020 & $19 . \therefore 1$ \\
\hline CRAB (NGC1952) & 5.5 & 22 & 42 & .506 & .993 & .887 & $6.4 x$ \\
\hline HER X1 & 17.0 & 35 & 75 & .024 & .029 & .760 & $28.16 i$ \\
\hline CYG X3 & 20.5 & 41 & 67 & .962 & .178 & $.75 \pi$ & 21)...': \\
\hline M31 (NGC224) & 0.7 & 41 & 53 & .512 & .814 & .233 & 10.1 .1 \\
\hline GEMINGA & 6.5 & 18 & 55 & .386 & .734 & .124 & 11...?! \\
\hline SS 433 & 19.2 & 41 & 66 & .707 & .213 & .035 & 19...1 \\
\hline $3 \mathrm{C} 273$ & 12.5 & 2 & 74 & .608 & .037 & .456 & $27.1: 1$ \\
\hline GALACTIC CENTER & 17.7 & -30 & 61 & .093 & .436 & .716 & 15.21 \\
\hline CAS A & 23.3 & 59 & 61 & .888 & .436 & .416 & 15.21 \\
\hline SCO X1 & 16.3 & -16 & 52 & .077 & .848 & .635 & 9.97 \\
\hline ALPHA CENTAURI & 14.6 & .61 & 55 & .929 & .734 & .398 & $11 . .: ! !$ \\
\hline maximum (IMB 1) & 1.2 & 26 & 64 & .467 & .293 & .003 & $17.6 ?$ \\
\hline
\end{tabular}

IMB 3. The list has eppeared in previous annual reports.

We have also used our two prong analysis program to search for candidates. In this method two distinct tracks must be found for an event to be a candidate. Since the event must be of low momentum the two tracks are fairly far apart and easily distinguished. The method is limited to the modes listed in table I.

\subsection{Point Source Search}

While the majority of the events collected in IMB come from atmospheric neutrino interactions, it is possible that a point source is also present. We have studied the 14 source directions examined in IMB 1 and have set limits on the possible excess neutrino flux that could come from these sources. A limit of about $10^{-2}$ neutrinos $/ \mathrm{cm}^{2} / \mathrm{sec}$ is obtained on sources in the $1 \mathrm{GeV}$ energy range. Table 2 lists the 14 sources studied and limits on the 


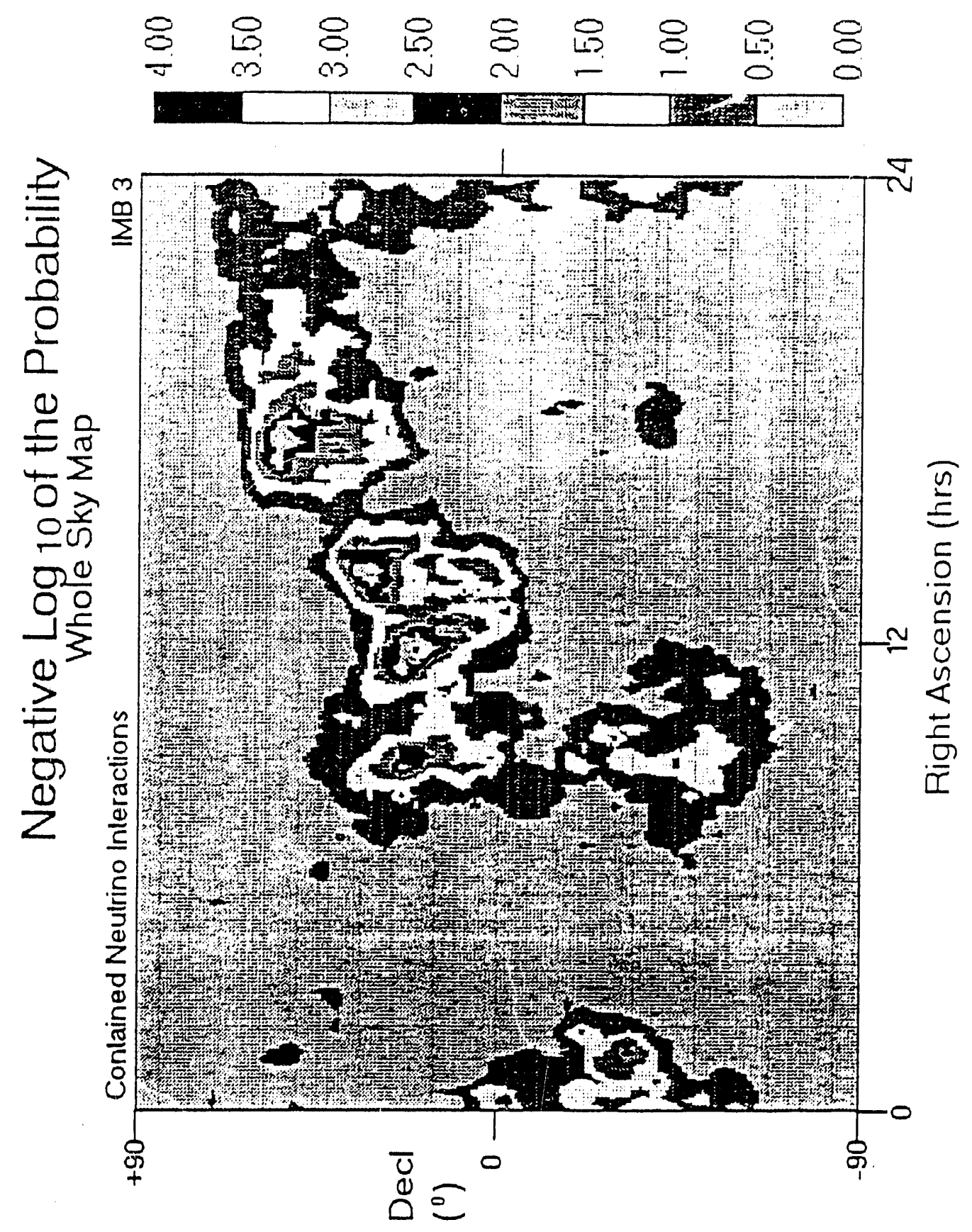

Figure 1: Sky map of contained IMB events. The negative log of the poisson probability is plotted. 
number of excess events. This same source list was studied using all 887 contained events and the 312 events classified as single track events with more than $500 \mathrm{MeV}$ of visible energy. For sources below the horizon, even part of the time, the upward muon sample was also searched. The only source with a consistently low probability of being a fluctuation is Hercules $\mathrm{X} 1$. Given the relatively crude search resolution of $30^{\circ}$ it would be hard to conclude this result is significant or is associated with that particular source. This source persists as the most significant one studied even in the upward muon sample. The upward muon sample represents muon neutrino interactions of high energy occurring in the earth below the detector. The upward muon sample does not overlap with the contained event sample but the normalization is made more difficult by the absence of uniform sky cover. Various ways of normalizing the data all lead to finding Hercules XI as the most significant source. 7 events are observed when less than 2 are expected. When the number of trials is considered this is still less than a 2 sigma result.

Beyond the search for the IMB source list two other searches have been made. Sky maps have been constructed using the event rates from a portion of the sky sampled at very close overlapping intervals. The poisson probabil. ity at every point can be calculated and a map constructed. Unfortunately it is often difficult to reasonably calculate the true significance of the highest point found on such a map. The most unlikely point on the map is located at 11.8 hours +15 degrees and has a poisson probability of $10^{-3.99}$. 68 events were observed where 42 were expected. But the map represents 4608 trials. When the energy cuts are applied this location is no longer the most significant. Instead a direction near 17.3 hours +52.5 degrees is.

At the risk of destroying the significance of any of the work done above we have also examined the 334 direction GRO target list for possible excesses. This list represents all possible high energy gamma ray sources to have been reported or even suspected. One might expect only about 334 times .05 of these sources to appear as statistical fluctuations upward. That is about 15 sources should be found to be high at the $5 \%$ level. We find 20 in the full contained sample and 10 in the cut high energy sample. Only 4 sources are found significant at the $5 \%$ level with the upward muon sample but only a subset of the sources can be sampled below the horizon. These numbers clearly indicate that the sky is not filled with sources that are bright compared to the atmospheric neutrino flux. Still the top candidate sources should be 
publicized in the hove that it might stimulate additional work.

The single most Important conclusion of this sectlon is that sources with fluxes as low as $10^{-2}$ neutrinos $/ \mathrm{cm}^{2} / \mathrm{sec}$ could be found with the IMIB detector. This is comlluaruble to the flux sensitivity in zome gamma ray energy bands.

\subsection{Underground Muons from Cygnus X3}

INB contlnues to tag and store the directions of all muons that trigger the detertor. Thio nrchivad mample can be searched if there is any indication that somelhing interesting could be found. Note that it is not continuously studied. We have searched in periods of about a week during June and July 1989, Augugt 1990 and January 14 to 28, 1991 where radio outburst have been noted. An excess of underground muons from the direction of Cygnus (ii) dullug llie Jumunry 1991 outburst han been reported by the Soudan II group. In particular they claim to see a significant flux excess on January 20 altd 22.

For the 1989 and 1990 outbursts we performed the standard Cygnus X3 phase analysis and found no enhancement at any phase bin. The data and background estimutes matched with good chl square. The 1989 result was published. We got the 1990 data too late to make it into the article on the 1098 datn. The flux limits from the source is estimated at $3 \times 10^{-111} \mathrm{~cm}^{-2}$ for the 1989 outburst and $3 \times 10^{-11} \mathrm{~cm}^{-2}$ for the 1990 outburst.

We have aloo searrhed for the DC excenn rmp!nt torl he Lhe Snillnn II group. Because of the brief nature of the reported underground signal (Two periods of one day each) it wasn't possible to achieve these high sensitivitieg. Ćygnus is only well visible for about 4 hours a day. For the day of January 22 at the IMB depth our flux limit was $7 \times 10^{-10} \mathrm{~cm}^{-2} \mathrm{gec}^{-1}$ at $90 \% \mathrm{c} .1$. The Soudan II result has been estimated at a flux of $8 \times 10^{-111} \mathrm{~cm}^{-1} \mathrm{sec}^{-1}$ but IMB is at a shallower depth ( 1570 mwe as compared to 2200 mwe for Soudan) so a higher flux would probably be called for. I'le limil lise Janulury 20 'th 1991 is $5 \times 10^{-111} \mathrm{~cm}^{-2} \mathrm{sec}^{-1}$. Both of these limits are in conflict with the Soudan result. 


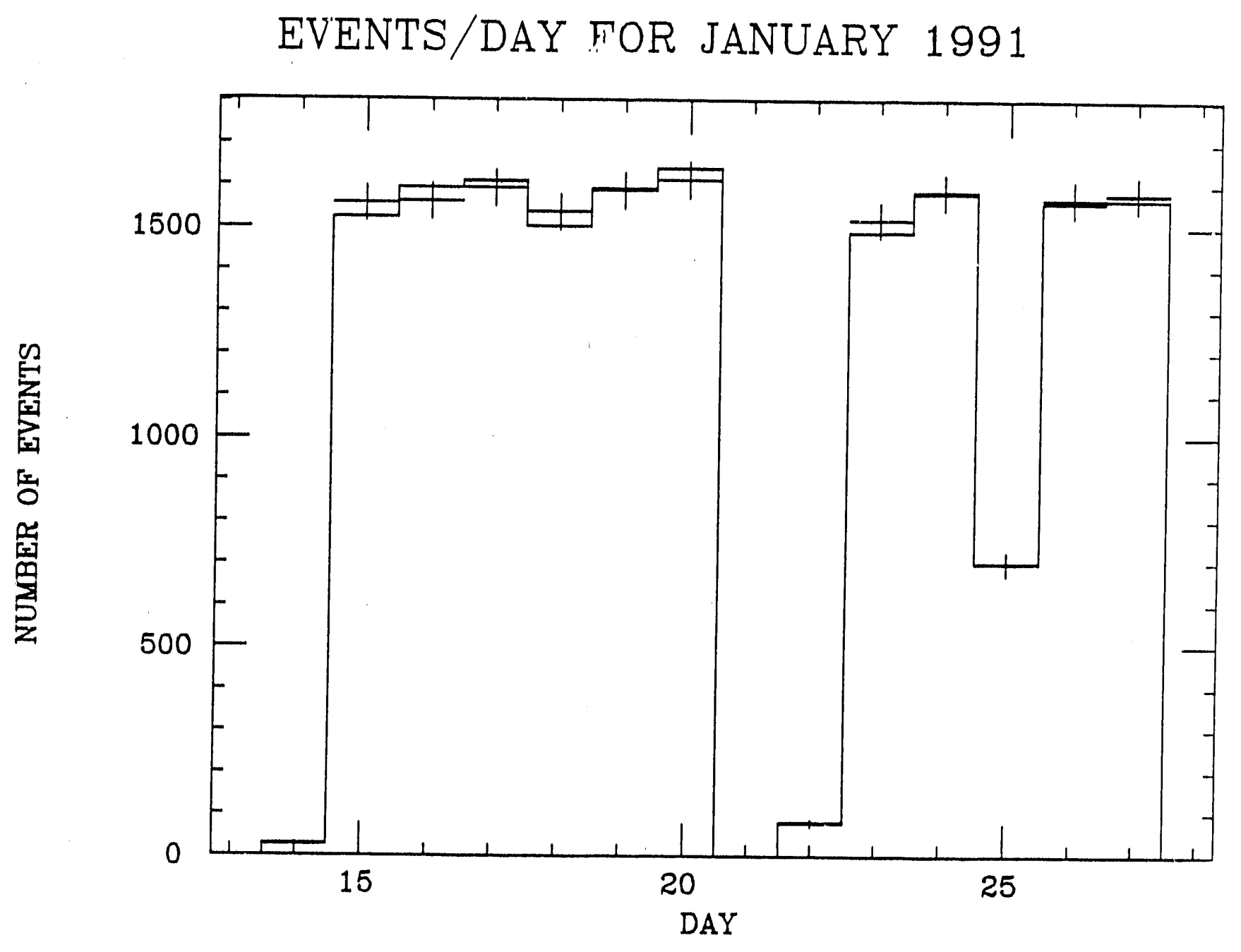

Figure 2: Search for DC excess from Cygnus X3 during the January 1991 radio outburst 
Table 3: Neutrinos from the Sun

\begin{tabular}{|c|c|c|c|c|c|c|}
\hline $\mathrm{C}$ & Energy & $\begin{array}{l}\text { Total } \\
\text { Events }\end{array}$ & $\begin{array}{r}\text { Events Near } \\
\text { the Sun }\end{array}$ & $\begin{array}{l}\text { Poisson } \\
\text { Prob. }\end{array}$ & ז90 & $\begin{array}{l}90 \% \text { (l) } \\
\text { Up Linl }\end{array}$ \\
\hline & $E_{\nu}>0$ & 677 & 54 & 0.090 & 0.031 & $\overline{19.81}$ \\
\hline case 1 & $1 \mathrm{GeV}<\mathrm{E}_{\nu}<2 \mathrm{GeV}$ & 182 & 11 & 0.560 & 0.036 & 6.211 \\
\hline case 2 & Upward Muons & 651 & 4 & 0.099 & 0.009 & 5.74 \\
\hline case 3 & $\mathrm{E}_{u}>2 \mathrm{GeV}$ & 24 & 2 & 0.219 & 0.184 & 4.114 \\
\hline
\end{tabular}

\subsection{Dark Matter}

It has been known for some time that dark matter may be detected indirectly through the production of energetic neutrinos from celestial objects. The process involves scattering on the matter of the sun or earth (or other celestial object). The subsequent loss of energy leads to gravitational trapping. Once trapped the concentration of dark matter particles will grow until the annihilation rate equals the accretion rate. This makes it easier to calculate the neutrino flux in the equilibrium limit. We note that the cross sections used to calculate the trapping rate are the same as those used in direct observation experiments. So our results can be compared with those experiments. Direct observation of dark matter does not depend on the annihilation cross section but in equilibrium our result doesn't either. In equilibrium the neutrino flux from the sun only depends on the capture rate since capture and annihilation rates are equal. So direct observation and indirect observation can be compared, unless the sun has not yet reached equilibrium, which is considered unlikely

The simplest signal to observe is an excess of neutrino flux in the direction of the earth or sun. The atmosphere provides an unavoiable neutrino background. The background also provides a convenient normalization. In particular measuring the ratio of the neutrino flux from the direction of the earth or sun relative to an off source region yields strong limits on the existence of dark matter in the solar vicinity. Olive and Srednicki have pursued this idea. In fact, they quote their theoretical predictions for a dark matter induced fiux relative to the atmospheric flux and suggest a quantitative test for significance as a function of the dark matter model. Table 3 lists the IMB limit for $\mathrm{r} 90$ computed using the Olive and Srednicki method.

Using their idea and the IMB data samples we has succeeded in ruling 
out all candidates of massive Majorana or Dirac neutrinos and all masses of scalar electron or muon neutrinos. and placing limits on candidates such as Higgsinos and photinos.

\subsection{Neutrino Oscillations}

Neutrino Oscillations can be studied to some extent, using atmospheric neutrinos. The atmosphere is a classical mixture of electron and muon neutrinos. Though they are not both present in equal proportions oscillations of electron neutrinos into muon neutrinos and vice versa are very difficult to study, in general. On the other hand a number of tests can be carried out that take advantage of the $10^{\top}$ meter path lengths possible with atmospheric neutrinos. In particular, we have a source distributed at distances varying from about $10 \mathrm{~km}$ to $10,000 \mathrm{~km}$.

Several tests can be performed. The overall rate of electron and muon neutrino interactions may be studied using identification methods developed and described in last years report. These analyses tend to indicate an excess of eleciron neutrinos, above expectations. But many of us suspect that the systematic error is about $15 \%$ which would eliminate the significance of the effect. In water detectors scattering, poor fits etc. all tend to smear the cherenkov image. Smeared images are taken to be electron induced. There is no compensating mechanism to misidentify electrons as muons. The significance of the Kamioka and IMB results on the overall electron to muon ratio should not be overestimated.

By comparing neutrinos that have travelled different distances a direct test of an $\mathrm{E} / \mathrm{L}$ dependence can be made. Such a test is sensitive to neutrino mass differences as low as $5 \times 10^{-3} \mathrm{eV}^{2}$. This test has been carried out with both the IMB 1 and IMB 3 data samples. Similar contours are obtained (Figure 3 ). The sensitivity is limited by the relatively poor statistics. Only single track events that contain a muon decay used in the sample. The limit must be considered a limit on muon to tau oscillations only. To study electron neutrino oscillations or muon - electron oscillations one must include matter effects in the earth. Beyond that the situation is made a bit more complicated. One must identify a clean sample of electron neutrino interactions in IMB. Combining such a sample with a numerical integration of matter effects 


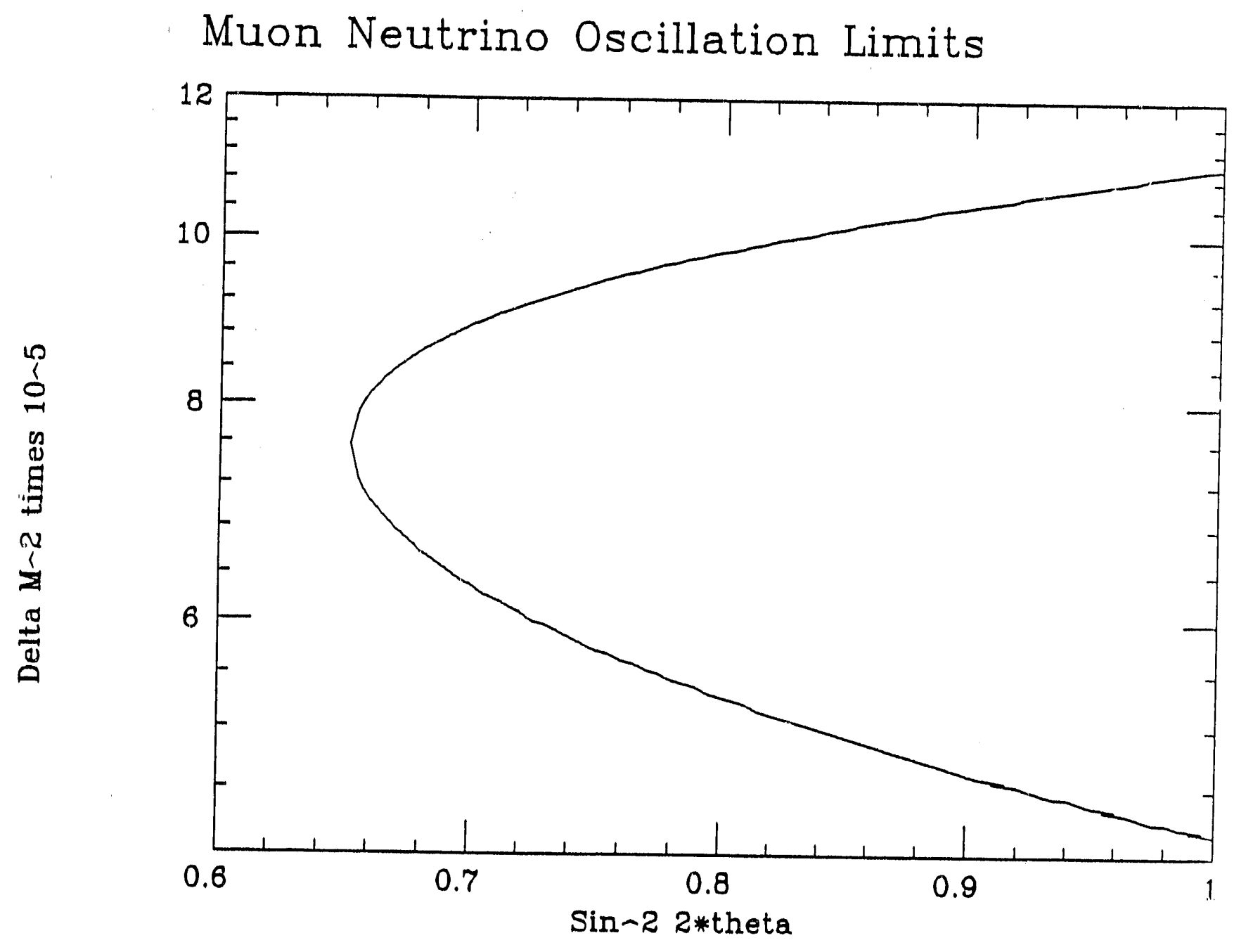

13

Figure 3: IMB Contained Nentrino ()scillation Limits 
would yield a limit on electron to tau oscillations if one assumed the muon neutrino were stable and didn't oscillate. Since the source is a mixture of electron and muon neutrinos it seems hard to disentangle the two oscillation rrobabilities. We did succeed in doing this a few years ago, at low energies. At neutrino energies below the muon neutrino threshold oscillations of muon neutrinos into electron neutrinos would produce a big enhancement. Such an enhancement was not seen and a good bit of the "Bethe" solution to the solar neutrino problem could be excluded.

Recently it has been realized that ihe oscillation limits on muon neutrinos could be extended upward in $\Delta m^{2}$ by using the upward muon sample. In general the upward muon sample is less relizble for studying oscillations since the energy is not measured and the distance does not vary much over most of the observable range (upward). Prior tests have compared the total rate observed with the total rate predicted. Limits on oscillations would follow if the observations were close to expectations. But the limits depended strongly on an assumed neutrino spectrum. Recently, by using a small sample of stopping upward muons a less model dependent limit has been achieved. One compares the upward stops, with the upward throughgoing muons. This result does depend on understanding $d E / d x$ energy losses in rock and the low energy end $(2-10 \mathrm{GeV})$ of the spectrum.

\subsection{The Future of IMB}

In May of 1991, following a serious leak it was decided to terminate operation of the IMB 3 detector. Hopefully this will result in a more rapid analysis of the data and the extraction of physics results. When the data through that date is finally analyzed we will have a total sample of more than 2000 events. I:imits on proton decay approaching $5 \times 10^{32}$ years will be reasonable.

IMB continues to suffer from very poor management. There is little reward for accomplishing physics. In general such work is met with severe criticism and rejection. For example, since IMB 1, no fewer than 5 people (Blewitt, Seidel, Mudan, Dye and Cady) have attempted to produce papers on proton decay. All were trashed. Our single paper on proton decay to $e^{+} \pi^{\prime \prime}$ was delayed for about 2 years because agreement could not be reached about why one event was not a candidate. Even though there was agreement that 
there were no candidates.

Given this environment there is very little motivation for young people to get involve and most of the more senior members are no longer actively pursuing IMB physics. This is unfortunate since the previous sections have demonstrated, we hope, the great scientific potential of the data already acquired.

\section{Meson Spectroscopy}

\subsection{Physics Motivation}

QCD, the theory of strong interactions, has bee: around for over 18 years. Most evidence in support of $Q C D$ has been indirect. While the quark structure of hadrons seems to be well born out by the quantum numbers and masses of the known hadrons, the evidence for gluons is much less precise. The value of $\mathrm{R}$ in $e^{+} e^{-}$scattering is taken as good evidence for 3 colors but does not manifest the gluons, since they are electrically neutral. 'The presence of gluons can be inferred from deep inelastic scattering, where it has been known since the early days that the charged parts of the proton do not carry all of the momentum. Three jet events and quark gluon plasma have been suggested as good evidence for gluons but to a large extent all they do is confirm the presence of glunn degrees of freedom while telling us very little about their couplings. What little evidence we have for gluons is an indication of additional degrees of freedom, beyond quarks, but not a clear indication of how these gluons interact.

Meson spectroscopy is known to be an ideal method to study gluon interactions. Mesons are simpler and lighter than baryons and the additional gluon degrees of freedom can modify the quantum numbers to values that can not be achieved with a quark-antiquark pair. That is mesons with quantum numbers $\mathrm{J}^{\prime \prime C}=0^{--}$or $0^{+-}$or $1^{-+}$or $2^{+-}$can be produced. These new nesons, with unusual quantum numbers have been given the name "exotics". The most exciting exotic, would be a particle composed of pure gluons, a glueball. Such a state is absolutely inevitable if hadrons are held together by a self coupling octet of gluons. To date, no conclusive evidence for glueballs 
has been found. Another form of exotic is the $q \bar{q} g$ bound state of quarks and gluons (where the $q \bar{q}$ is in a color octet). Such states are known as "hybrids". The existence of this class of exotics is inevitable in QCD as presently formulated. Color neutral states are not confined and gluons behave like an octet under the $\mathrm{SU}(3)$ color group. If both of these statements are true color singlets can be constructed out of all glue or out of an octet combination of $q$ and $\bar{q}$ and a gluon. These may have exotic quantum numbers. One can also expect that the masses of these non-quark objects would be relatively light. The expected masses are well within the range of the current experiment.

The GAMS group has done extensive work looking for new mesons that decay to all neutral final states. A number of interesting candidates have been found. GAMS has found a $\mathrm{J}^{P C}=1^{-+}$candidate at a mass of $1405 \mathrm{MeV}$. The state decays in $\div 0 \eta \pi^{\cup}$. Since it has isospin 1 it can not be a glueball but may be a hybrid or a $q q \bar{q} \bar{q}$ state. These two possibilities may be distinguished by the relative branching ratio to $\eta \pi^{U}$ and $\eta^{\prime} \pi^{U}$. GAMS has also reported a scalar meson $\mathrm{G}(1590)$ decaying into $\eta \eta$ and $\eta \eta^{\prime}$. Other states in this mass region which have been studied with radiative $J / \psi$ decays include the $\eta(1440)$ and the $f_{2}(1750)$.

Even the conventional $q \bar{q}$ sector needs to be clarified. Many standard states have masses in the region of the $\eta(1440)$ glueball randidate, such as the axial vector state $f_{1}(1420)$. A map of the light meson nonets is needed against which one can compare exotic candidates. The isoscalar and isovector components of the still missing nonet ${ }^{3} D_{2}$ are expected to decay predominantly into $\eta \omega$ and $\pi \omega$ respectively.

All of these reasons point toward the need for an exhaustive study of charged and neutral final states in moderate mass, nonflavored mesons.

\subsection{Brookaven E852}

A collaboration of Brookhaven National Laboratory, Indiana University, University of Louisville, Southeastern Massachusetts University, the University of Notre Dame, Moscow State University and IHEP Serpukhov has been formed to study these questions at the Brookhaven AGS. A. Dzierba and S.U. Chung are cospokesmen for this experiment, number 852 .

The objective of Brookhaven experiment 852 is to study mesons with 

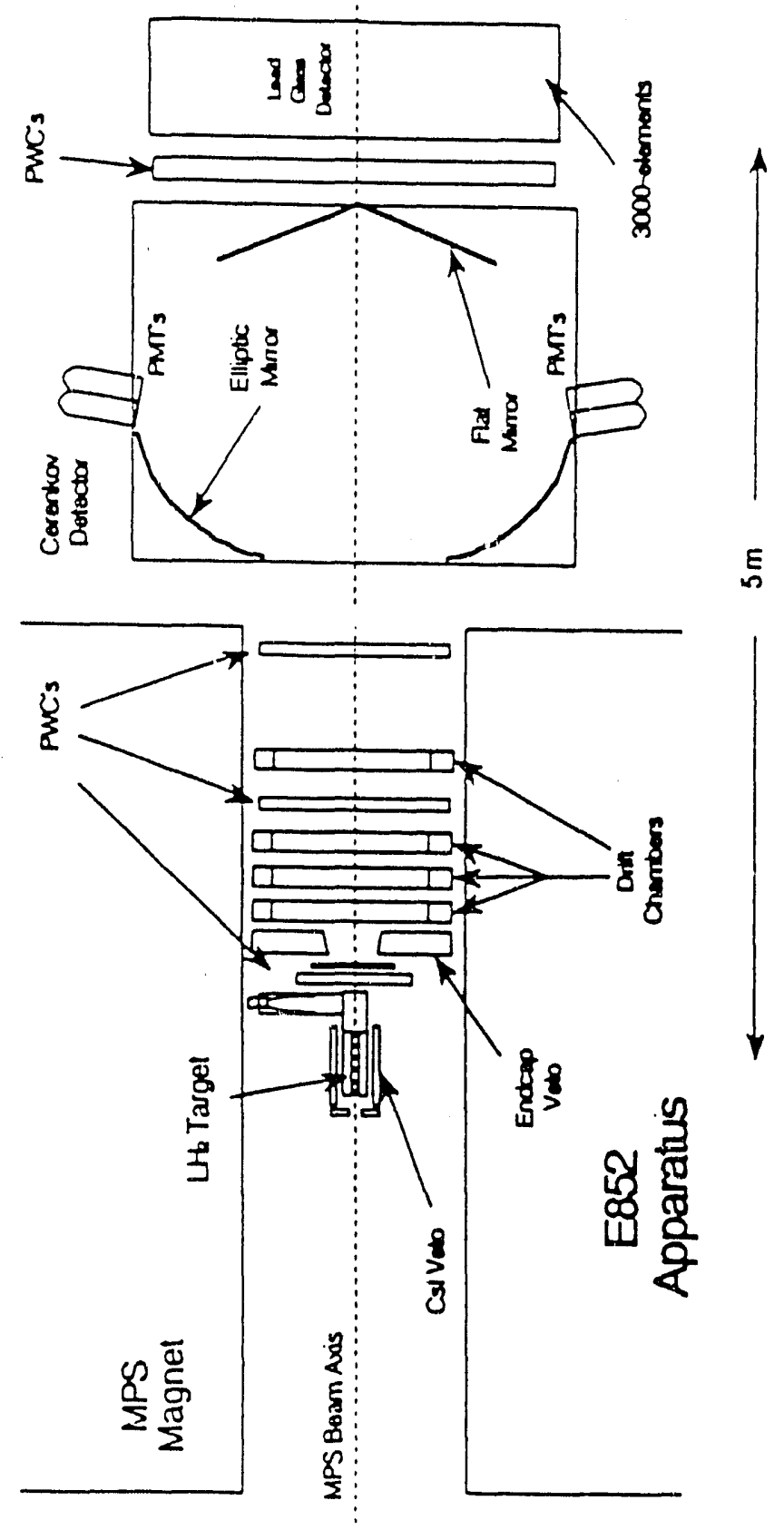

17

Figure 4: Brookhaven Experiment 852. 
masses below about $3000 \mathrm{MeV}$. The experiment was inspired by the success of the GAMS collaboration in studying all neutral final states. Our detector goes beyond the original aims of GAMS in that we will have both charged and - eutral detection and Cherenkov counters for kaon identification. Ultimately we hope that this study will shed some light on the nature of glueballs and exotic mesons which are expected in QCD but not in a simple quark model.

Recent experiments which bear on this question use a variety of techniques: $J / \psi$ radiative decays (Mark II, Crystal Ball, Mark III and DM2 experiments); $\gamma \gamma$ interactions (Crystal Ball and Cello experiments); central hadroproduction (WA76); peripheral hadroproduction (Notre Dame experiment BNL E769, BNL E771, LASS at SLAC and GAMS at Cern and Serpukhov); and $p \bar{p}$ annihilation (Lear experiments Asierix, Crystal Barrel and Obelix). Each of these experiments has its strong points, and it is only with data from many sources that the nature of the meson spectrum will be understood in detail.

Operating at Broukhaven gives us an adcitional advantage in that the amplitude analysis can be carried out over a much larger solid angle. The detector granularity is not nearly as significant as at high energies. Production cross sections to specific final states are much higher than at the energies used by GAMS at CERN or Serpukhov for masses most favored as glueball and exotic candidates.

The experiment involves major modifications to the BNL MPS to enable it to study all neutral final states. It is deemed prudent to maintain the charged particle detection so that both charged and neutral final states can be observed in the same experiment. Major components of the detector include the lead glass wall 2.84 meters wide by 1.72 meters high, with 3000 4 by $4 \mathrm{~cm}$ blocks. These blocks are, in fact, being produced by the same soviet factory that produced the GAMS detectors. Resolution of this lead giass wall is estimated to be about $1 \mathrm{~mm}$ in position and about $(2+5.5 / \sqrt{E})$ $\%$ in energy. Beam tests indicate that it might even be somewhat better. Simulations indicate that, while very good, it is the energy resolution that will ultimately limit the experiment. Such an array has good acceptance for both charged and neutral final states for masses well above $2 \mathrm{GeV}$

Tn determine the exact nature of the inclusive final state a Cesium Iodide. target veto box and a downstream endcap array. of lead scintillator have been added to veto stray photons that come, for example, from the decay 
of a $\Delta^{\prime \prime}$. The presence of soft photons in the final state can change the quantum numbers assigned to the produced state. With the CsI veto events of the form $\pi^{-} p \rightarrow X N^{*}$ can be tagged and removed by the detection of the photons from the $\pi^{\prime \prime}$ decay of the $N^{*}$. A lead scintillator sandwich will be used to tag more energetic, forward going photons that would miss the CsI and the lead glass wall. Care is needed in the design and placement of this downstream array to prevent it from restricting the charged particle tracking capabilities of the experiment. Existing tracking chambers will be used to reconstruct charged particles. Some PWC's are planned to get rapid information on charged particle multiplicities for the trigger. A segmented Cerenkov counter will be added to do pion and kaon separation. A hydrogen target will be used but it will be placed at the center of the MPS magnet. Tracking near the target, for proton recoils, is accomplished by a three layer set of cylindrical chambers. There has even been some discussion of running with a $\pi^{+}$beam on a deuterium target to study the analogous initial state of $\pi^{+} n$. This has the advantage that it should produce the same meson sper'-um but often have a trackable proton recoiling from the target. Some resolution may be lost because of the deuterium Fermi motion.

The combined charged plus all neutral analysis permits careful measure. ment of isomultiplets such as the $\eta \pi^{U} \mathrm{P}$-wave state at $1405 \mathrm{MeV}$ reported by GAMS. If confirmed (in the charged channels) this state is clearly exotic having $\mathrm{J}^{\prime} \mathrm{C}=1^{-+}$. The combined charged plus neutral detection is also complimentary for example, in the decay of the $\eta^{\prime}$ into $\eta \pi \pi$ where detection of low energy charged pions is complementary to the detection of the high energy photons from the $\pi^{\mathrm{U}}$ decays.

We are scheduled to start data taking in January of 1993. We expect to continue acquiring data over a period of of about 3 years. This schedule is realistic even in the case of possible equipment budget shortfalls since we would attempt to run on all neutral final states (a la GAMS) if the full tracking and Cherenkov is not available on time.

The Brookhaven lab management is fully committed to experiment 852 . In fact, they see it as the first experiment in a major program in non-flavored meson spectroscopy. As mentioned the Brookhaven AGS is ideally suited for this kind of physics. 


\subsection{Responsibilities}

The principal hardware contribution of the Notre Dame contingent is the Cesium Iodide target veto. This contract's responsibilities include the electronics for the veto, the calibration and monitoring system for the CsI, the target region mechanical design and event simulation and reconstruction. We will assist in acquisition and acceptance tests for the Cesium Iodide.

We have been influential in pushing the preamp threshold on the CsI to well below $1 \mathrm{MeV}$. While this is a bit below the threshold needed for the physics it provides an easy monitoring and calibration method and permits us to test and study the final detector in the lab without the need for additional beam tests. A low threshold may also make it possible to use this detector for a number of other physics objectives. Since the CsI is only $7.5 \mathrm{~cm}$ thick it cannot be used for accurate energy measurements above a few hundred $\mathrm{MeV}$.

The target region design is being worked on jointly with Neal Cason of the NSF group.

\subsection{Progress to Date}

The overall design of the detector is finished and we are in the process of doing the mechanical and electronic designs for specific components. We have acquired 9 CsI blocks from 3 manufacturers, several different silicon photodiodes and 5 different preamp designs. CsI was chosen for the target veto since it is highly efficient, fairly compact, well understood and readily available. The silicon photodiode readout is dictated by the positioning of the veto box around the hydrogen target at the center of the MPS magnet. We anticipate operating the magnet at about 10 kilogauss during normal running.

We have tried to develop resonable performance criteria for the CsI before requesting bids for the full order. We were concerned about total light output, afterglow and noise. Since we are operating in a fixed target hadron experiment some of our criteria differ from that used by recent electron collider experiments. In particular we anticipate much higher rates, as much as $1 \mathrm{kHz}$, and would like to easily see much lower signals, below $10 \mathrm{MeV}$.

Major progress was made during a Brookhaven beam test in May of 1991. 


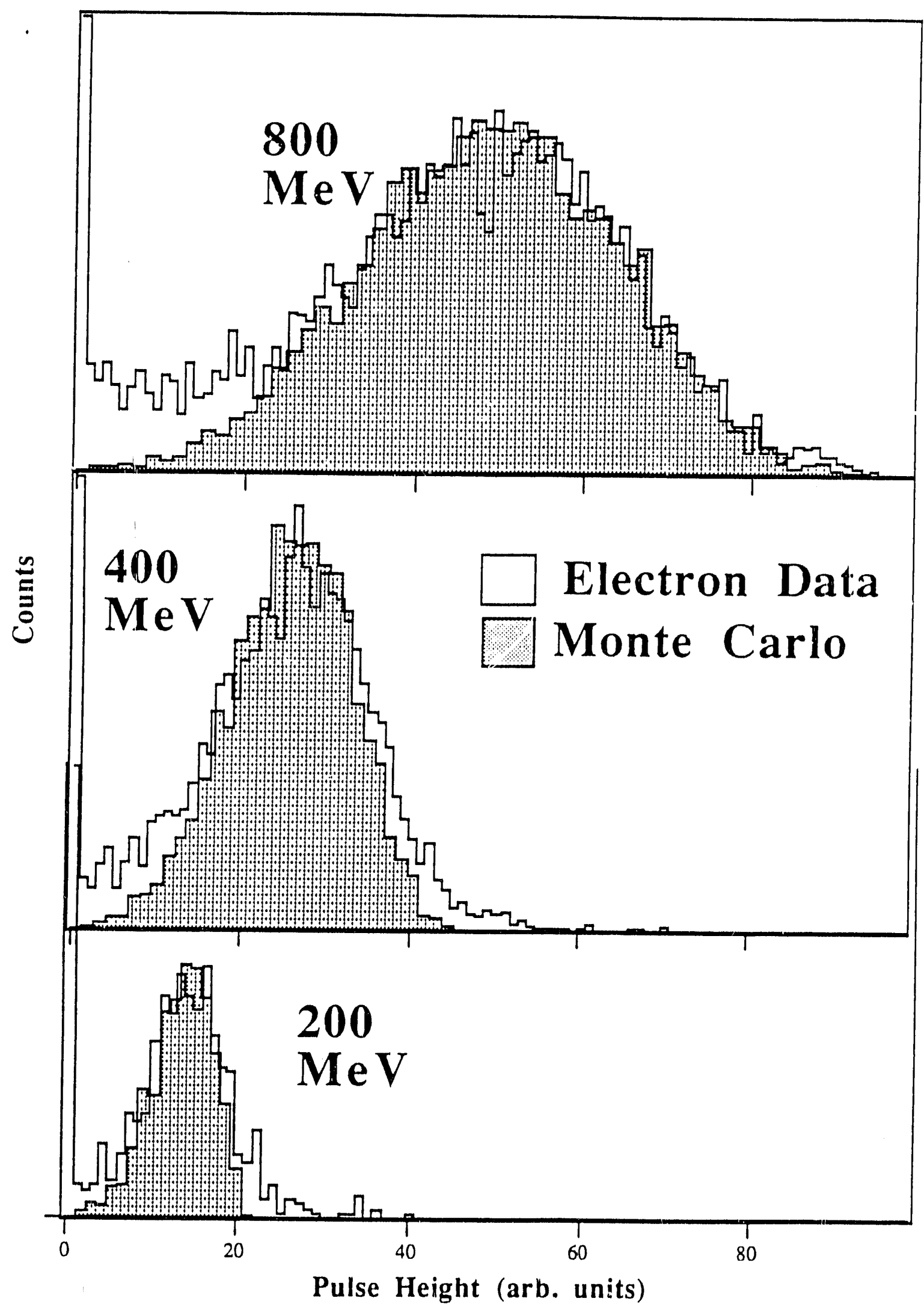

Figure 5: CsI beam test data compared to Nonte Carlo 


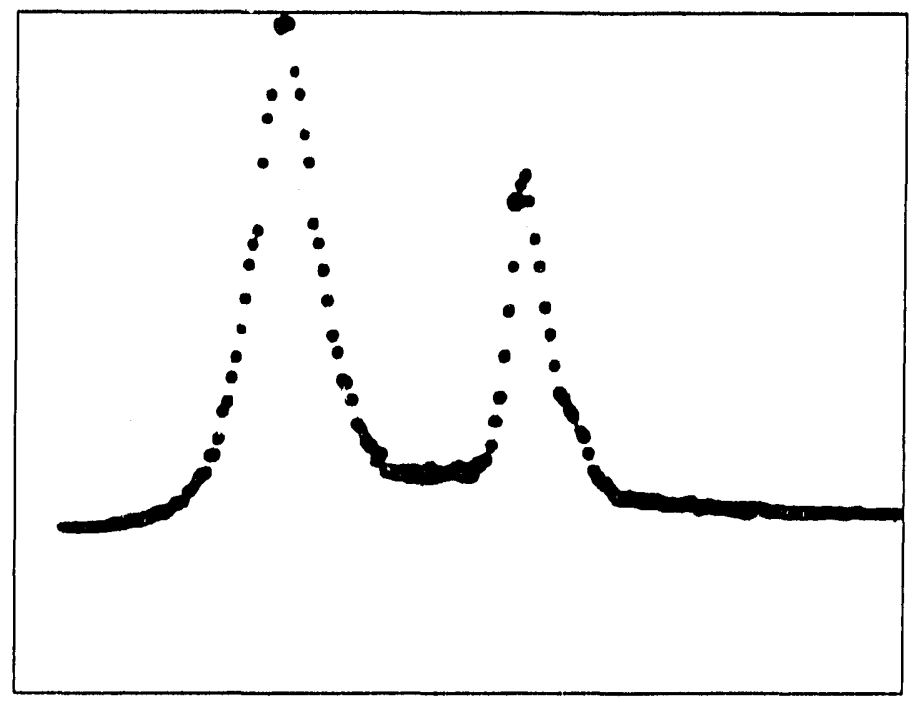

Figure 6: Cobalt 1.2 MeV gamma peak in CsI with our readout electronics

We were able to acquire photodiode preamps with less than $500 \mathrm{keV}$ noise, in our application. We tested in an electron beam down to $200 \mathrm{MeV}$ and found performance to be only slightly poorer than predicted by our simulations, Figure 5. We also ran at 400,800 and $1000 \mathrm{MeV}$. Resolution does not appear to be very gond in these plots but this is expected because the blocks are only $7.5 \mathrm{~cm}$ thick (4 radiation lengths) and a good deal of signal is lost out the back and sides. Back in the lab at ND we hare been able to observe the 1.173 and $1.333 \mathrm{MeV}$ lines of Cobalt-60, Figure 6. From the figure it should be clear that our operating threshold could be below $500 \mathrm{keV}$. We will use the Cobalt lines for both acceptance tests for the CsI and for monitoring the performance of the detector in situ.

Mechanical design and supports are coming along well. The CsI array alone weighs about 200 pounds. The preamps will be mounted on the back in a lighi proof box. CsI is slightly hygroscopic so we need a reasonably well sealed environment. At present we expect the crystals to be mounted inside a sealed cylindrical aluminum box cushioned by plastic foam so that 
the crystals are not under stress. Dual photodiodes and preamps are planned for reliability since access will be restricted in this region inside the magnet near a hydrogen target. Cabling will come through the outside cylindrical face to avoid interference with the target tracking chamber and hydrogen plumbing.

As presently designed, most of the additional electronics in the target region will be passive to give it high reliability. The very slow speed of CsI and long time constants on the preamps have presented some design problems but these have been deali with in a manner that preserves our sensitivity giving us the information that we need: the pulse height information, and a faster signal that can be used for a veto.

\section{$4 \quad$ SSC Ventures}

Recently the SSC organization has devoted its efforts on the review and approval of "Major Detectors". There can and should be a role for smaller SSC experiments. We are looking at the design of a possible detector that concentrates on observing electromagnetic decays of heavy particles. The detector is inspired by the $\mathrm{ZZ}$ decay of the Higgs but could be used for other searches. The idea is to trigger on Z's. E'ven in the presence of a substantial hadronic reaction rate the $Z$ decay should be observable as very large electromagnetic energy deposition in the central region. Such a large electromagnetic shower, at least a pair of large electromagnetic showers can be distinguished from the general hadronic background. Most energetic hadronic reactions will fill the regions of high rapidity. At the SSC those regions represent a small portion of the available solid angle. Our idea is to instrument only the central $90 \%$ of the solid angle and so to remove a large and dangerous part of the background. The device is a physically small segmented electromagnetic calorimeter.

The concept is to lonk for a very distinctive signatire in the presence of the expected hadronic background. One doesn't need to use fast techniques to beat the background. Since these will ultimately fail at high luminosities anyway. Instead the signal should be found superimposed on the background, including the background coming from multiple interactions per crossing. 


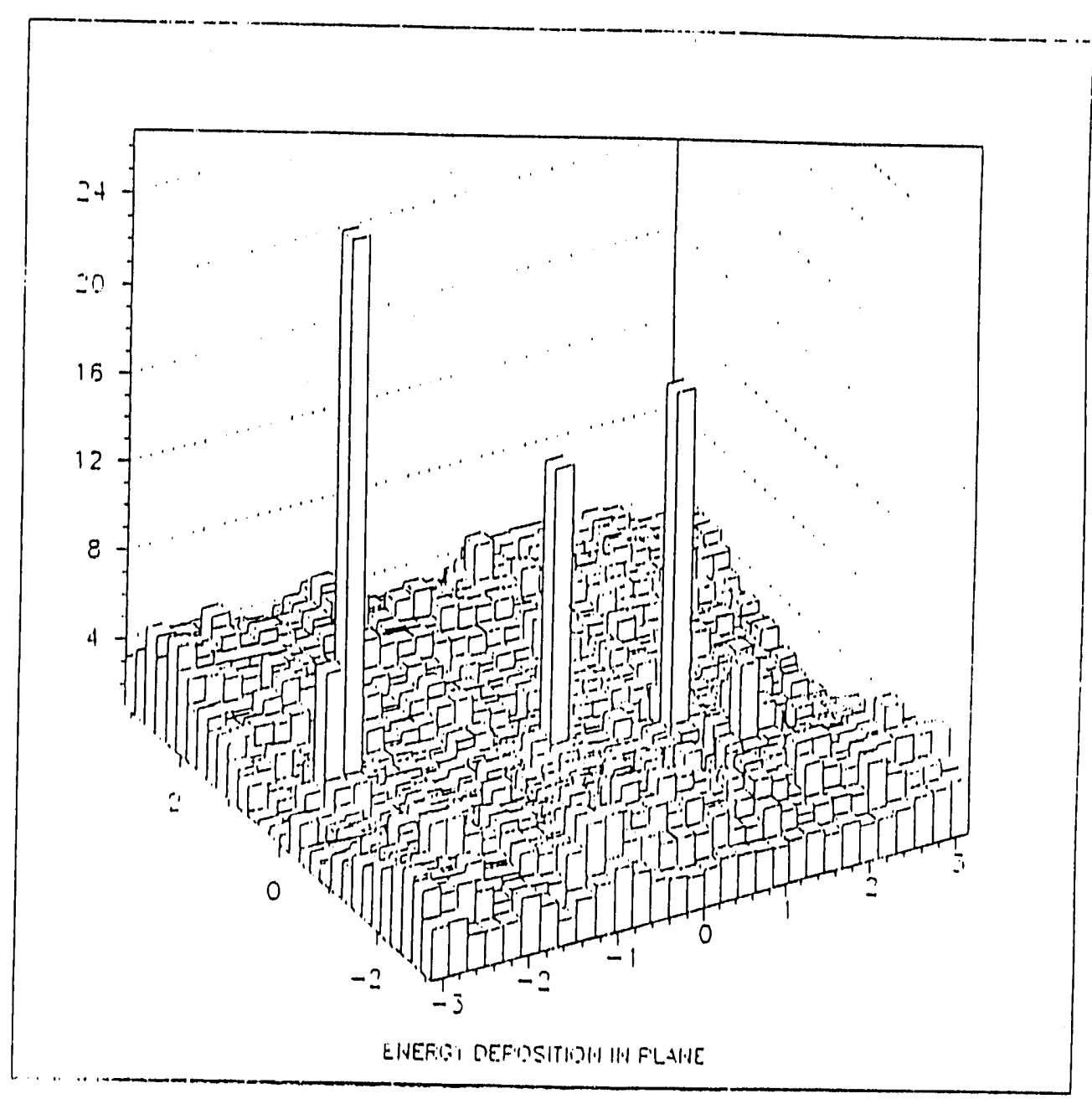

Figure 7: A simulated Higgs decay in the presence of 100 minimum bias background events. Note the clear peaks for the 4 electrons. 
In that respect the device should be able to use all of the available SSC luminosity at any given time.

This concept scales well with luminosity. In the early days the SSC luminosity will be low. Consequently searches will be limited to lighter masses. The search can be extended to highe: masses as the luminosity rises. Even though the background will increase with luminosity, the distinctive signature of the massive particle will become easier to distinguish from background as its mass rises.

We are continuing to study this concept. The SSC organization has indicated that it is still too soon to officially consider small detectors. The lab has made it clear that on the order of $\$ 100$ million will be made available for such ventures. A rough estimate of cost for our device is much less than a fraction of the funds to be allocated. 

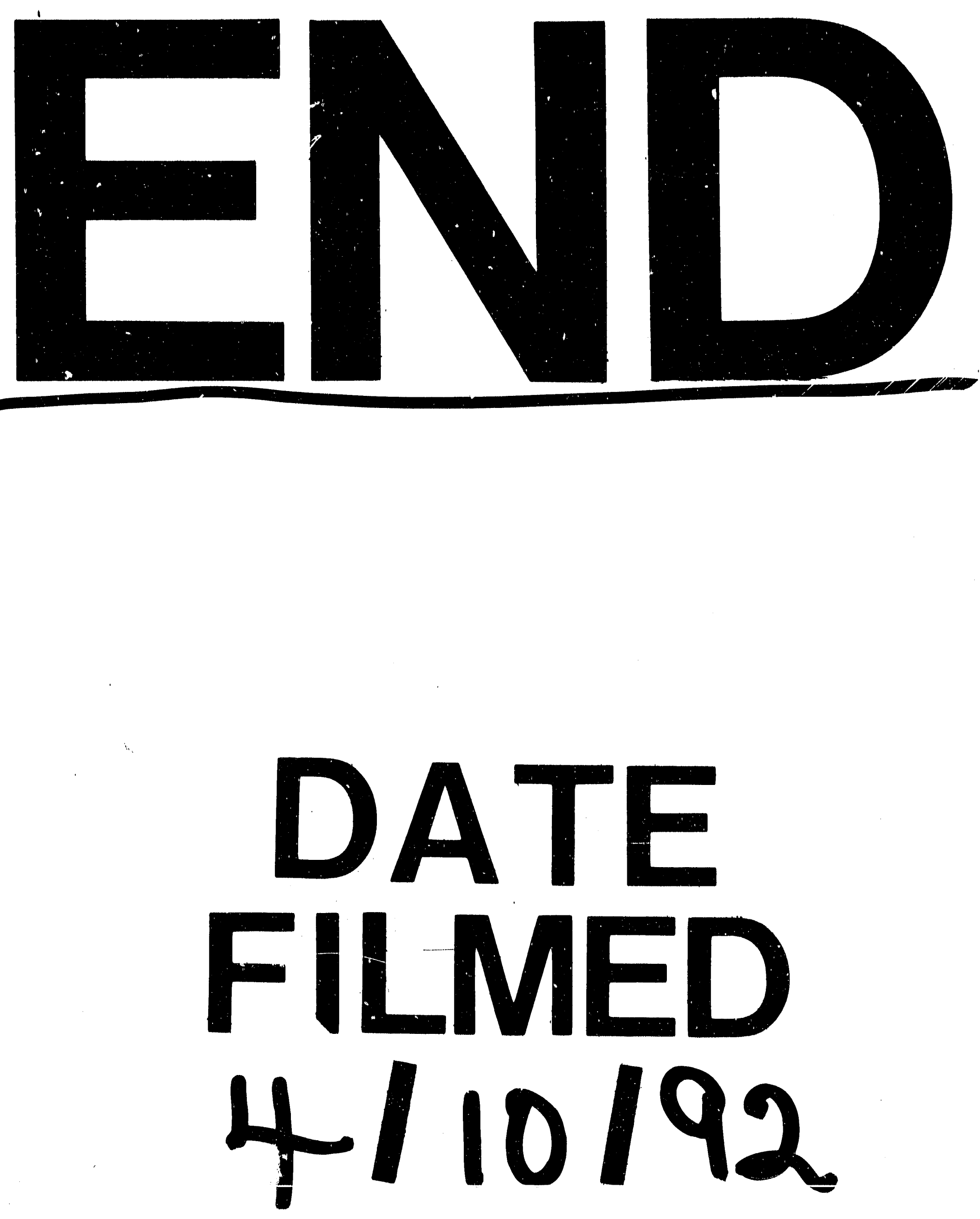
1

" 